E.L.U.A., 1, 1983, págs. 89-112

\title{
NUEVAS DISTINCIONES CONCEPTUALES EN GRAMÁTICA GENERATIVA Y ALGUNAS OBSERVACIONES SOBRE SINTAXIS Y DISCURSO
}

\author{
Violeta Demonte \\ (Universidad Autónoma de Madrid)
}

\section{INTRODUCCIÓN}

Creo que no es inadecuado afirmar que uno de los factores que puede haber incidido más positivamente en el desarrollo considerable que la gramática generativa ha sufrido durante los últimos años es su capacidad para reflexionar sobre sus propios supuestos básicos y para incorporar, a través de ellos, tanto las objeciones que se le oponían como los nuevos problemas relevantes que aparecian en el seno de la ciencia lingüistica.

Por eso, el propósito inicial de este trabajo es presentar, de una manera más especulativa que técnica, algunas distinciones metodológicas fundamentales o nuevos conceptos teóricos que bien han surgido como novedad, bien se ha explicitado y clarificado algo más a lo largo del período de gestación de la actual «Teoría de la rección y el ligamiento».

Una de esas distinciones concierne a las relaciones y diferencias entre las así llamadas gramáticas de la oración y gramáticas del discurso. De estas diferencias y relaciones me ocuparé doblemente en un segundo momento de esta exposición. En primer lugar, intentaré analizar la interacción entre dos tipos de elisiones: elisiones de la gramática oracional y elisiones del discurso y, como consecuencia de ese análisis —que tiene su base teórica en Williams (1977)—, sugeriré que las re- 
glas de discurso se aplican después de las reglas oracionales. Intentaré mostrar brevemente, por último, que la gramática generativa oracional ha hecho avances interesantes en la caracterización de diversos fenómenos que algunos autores consideran típicos del ámbito del discurso. Me referiré en particular al problema de las relaciones tema-rema en la estructura oracional.

\section{NUEVAS DISTINCIONES CONCEPTUALES Y SU TRASCENDENCIA METODOLÓGICA}

En efecto, como acabo de señalar, la teoría generativa ha ampliado y refinado sustancialmente en la última década el corpus de presupuestos generales o ideas directrices relativas a qué debe caracterizar una teoría del lenguaje humano. Este enriquecimiento de sus presupuestos filosóficos, por otra parte, es paralelo e interdependiente con un nada trivial desarrollo de la teoría gramatical. La confluencia de ambos procesos nos permite afirmar, en mi opinión, que está ahora más claro qué es lo que quiere y puede hacer, y qué no puede hacer, una gramática generativa.

Ahora bien, para delinear la génesis de estos procesos conviene señalar, en primer lugar, que aunque la distinción entre "competencia" y "actuación", introducida por Chomsky en su trabajo capital de 1965, haya sido necesaria e incluso decisiva para el desarrollo de las disciplinas interesadas en las aptitudes cognitivas de los seres humanos, no obstante, quizá haya podido resultar escasa para conducir el trabajo concreto, o, vista desde el ángulo opuesto, como abstracción de los rasgos fundamentales de los dispositivos técnicos y de los tipos de análisis que pueden llevarse a cabo por medio de una gramática generativa de las lenguas humanas.

Tanto la limitación de esta distinción primera como las discusiones y críticas a que dieron lugar la teoría estándar y la teoría ampliada han conducido a que hayan aparecido nuevas distinciones conceptuales y nuevos presupuestos teóricos sobre los cuales se asienta hoy el trabajo de elaboración de la teoría de la gramática y el desarrollo concreto de la misma. Las más importantes de estas nuevas distinciones son, a mi juicio, las siguientes: la introducción de la noción de "gramática nuclear" complementada por la de "gramática periférica", la diferenciación, dentro de la gramática nuclear, entre «principios" y "parámetros", el paso - en opinión de algunos analistas - de una "teoría derivacional» a una "teoría representacional» y el perfeccionamiento de la distinción entre "gramática de la oración" y "gramática del discurso". Pero veamos estas consideraciones con algo más de detalle. 
1.1. Distinción conceptual crucial para la metateoría e importante para el desarrollo técnico es la oposición entre gramática nuclear [core grammar] y gramática periférica. Hablando laxamente, dicha distinción puede conectarse con la que se establece entre "gramática universal» y "gramática particular" en tanto en cuanto, según Chomsky (1981 a), las gramáticas nucleares sólo pueden determinarse una vez que se han fijado los parámetros de la gramática universal. No obstante, una indicación de que esa conexión no es absoluta la proporciona la afirmación de Chomsky de que uen general, dos teorias distintas de la gramática universal (GU) delimitarán tanto la gramática nuclear como la periferia marcada, asi como las opciones dentro de la teoría de la gramática nuclear" (Chomsky, 1981 b, pág. 127). Pero la sugerencia contraria de que hay una importante relación entre periferia y lengua histórica aparece unas líneas más abajo en ese mismo texto de Chomsky: «En última instancia, las respuestas a las preguntas de la teoria del marcado provendrán del estudio en profundidad de las lenguas particulares dentro de una teoria de la GU fundada en principios, combinado con el tipo de enfoque exhaustivo que se ilustra en trabajos como los de Keenan" (op. cit. pág. 127); y en la afirmación de que «no es esperable que lo que llamamos "lenguas", "dialectos" o incluso «idiolectos" se conformen precisamente o ni tan siquiera aproximadamente a los sistemas determinados en la fijación de los parámetros de la GU. Esto sólo se da en condiciones muy idealizadas que nunca existen, de hecho, en el mundo real de las comunidades de habla heterogéneas" (Chomsky, 1981 a, pág. 8). (Esto parece sugerir, asimismo - y dicho sea de pasoque Chomsky concibe alguna intersección entre el estudio de los universales de la estructura superficial, que persiguen lingüistas como Comrie (1981), y su propia búsqueda de universales abstractos. Aquéllos podrían servir para delimitar la gramática marcada).

Ahora bien, en una aproximación más estricta, ese núcleo y esa periferia corresponden, respectivamente, al conjunto de elementos y construcciones no marcados que podemos esperar encontrar posiblemente en todas las lenguas y que parecen ser óptimamente accesibles desde el punto de vista del aprendizaje, y al conjunto de elementos y construcciones marcados que aparecen debido a la relajación de algunas condiciones de la gramática nuclear, a través de procesos de analogía, o por la adopción de préstamos, invenciones o reliquias históricas, entre otras posibles razones.

Independientemente de cuál sea el tipo de teoría de la marca sobre el cual repose la sintaxis - cuestión que por cierto no parece haberse debatido seriamente (cfr. Milner, 1982)- el concepto de marca empleado dentro de la concepción chomskiana de la gramática universal 
sirve, al igual que en fonología, tanto para fundar una teoría universal de las excepciones cuanto para sentar las bases de una teoría también universal de la comparación entre las lenguas. Con otras palabras: la distinción marcado-no marcado no solamente delimita lo general y extendido frente a lo particular e idiosincrático sino que permite hacer predicciones acerca de procesos posibles en lenguas próximas y conduce, asimismo, a un refinamiento de la noción de gramaticalidad.

Así, por ejemplo, y para volver sobre un problema estudiado por Milner (1982), el enunciado distribucional que afirma que en las oraciones finitas el sujeto es generalmente no-nulo mientras que en las nofinitas es por lo común nulo - una vez reformulado apropiadamente conforme a las nociones de marcado y no marcado-, permitiría, por una parte, explicar los rasgos especiales y la peculiar distribución de las escasas construcciones en las que un infinitivo va acompañado de sujeto en castellano (oraciones como Al salir el sol, cantaron los pájaros frente a *Al el sol salir cantaron los pájaros para las que podría postularse (cfr. Chomsky, 1982), si se cumplen otros supuestos, que actúa una regla marcada, excepcional, de asignación de caso) y, por otra, establecer una comparación entre las lenguas romances en las que el caso menos marcado sería el de aquéllas (si es que existen) en que no hay infinitivos con sujeto y el más marcado el de una lengua como el portugués en la cual los infinitivos pueden conjugarse.

Pasando a otro tipo de problemas, la introducción de la distinción marcado-no marcado (o la de gramática nuclear frente a gramática periférica) en una teoría con un alto grado de abstracción teórica permite también tratar de una manera más sofisticada los datos primarios recibidos de los hablantes, esto es, sus juicios de aceptabilidad. En un interesante trabajo sobre el estilo galileano de la investigación lingüística Rudolf Botha (Botha, 1982) pone de manifiesto que uno de los cambios existentes entre cómo se concibe la teoria en la exposición de «On binding» y en los momentos posteriores que anticipan a Chomsky (1981 a) consiste en que en este nuevo sistema ya no se entiende como en aquél que la aceptabilidad / inaceptabilidad de las oraciones deba ser un reflejo directo y correcto de su gramaticalidad / agramaticalidad. Más precisamente, este reflejo inmediato sólo podría darse en las estructuras no marcadas.

En efecto (Chomsky, 1981 b) sugiere ya que el carácter marginal, la dudosa aceptabilidad, de algunas construcciones puede deberse a que tengan un carácter marcado. Cinque (1982), por otra parte, utiliza inteligentemente la distinción marcado-no marcado para caracterizar ciertas propiedades estilísticas de las relativas italianas. Este lingüista desarrolla un exhaustivo análisis de las relativas restrictivas y apositivas 
del italiano en el cual hace intervenir cuatro principios que constituyen el núcleo no marcado de la gramática de la relativización. Ahora bien, las relativas marcadas estilisticamente, del tipo de Pedro, la hija del cual te saludó, es buen amigo, sólo podrian ser generadas por la gramática si se añaden a la misma dos principios suplementarios. Si consideramos, dice Cinque, que «el primer conjunto de principios comprende el núcleo no marcado de la gramática y el segundo una opción más marcada, disponible (a más alto precio) por la gramática, podemos deducir de una manera directa la diferencia de estilo observada si suponemos que el marcado estilistico es un efecto patente de la utilización de un aparato teórico más costoso" (Cinque, 1982; pág. 261, subrayado mío.- V.D.).

No me extenderé más sobre esta nueva distinción que podría merecer ella sola un trabajo independiente pero no quiero dejar de insistir en la relevancia que la misma puede tener si se articula inteligentemente con otros elementos de la teoría y no se emplea como cajón de sastre sino como concepto con capacidad explicativa.

1.2. Una importante idea que aparece estrechamente ligada a la distinción entre núcleo y periferia es la de que todos los elementos de los dos subsistemas de la gramática universal (el subsistema de las reglas y el de los principios (cfr. Demonte, 1983 b), en especial los del subsistema de principios, están sometidos a una cierta variación paramétrica. Esto significa, dicho brevemente, que será posible asignar a aquéllos un determinado valor a lo largo de una escala o, con otras palabras, que los principios que pertenecen al núcleo gramatical podrán encontrarse, no encontrarse o formularse de distinta manera, en las lenguas particulares. La cuestión, pongamos por caso, de qué es un nudo cíclico operativo para el "principio de subyacencia" (la restricción de localidad que constriñe la aplicación de la regla "Muévase $\alpha »)$ parece que es un principio parametrizable. Así, Rizzi (1982) muestra que en italiano el nudo cíclico que debe ser tenido en cuenta por dicho principio es 0 en vez de 0 . Según Kayne (1983), asimismo, la asignación de caso abstracto es también variable según parámetros. Kayne postula que en algunas lenguas, por ejemplo en el inglés, las preposiciones asignan caso objetivo y no caso oblicuo como sucede en otras. Lo más importante, sin embargo, de la articulación de la idea de variación paramétrica es que permite avanzar en el conocimiento de tipologías lingüisticas en la medida en que los parámetros se entiendan como 
racimos de propiedades determinados por una propiedad superior. Señala Chomsky, en efecto, que podemos advertir que existen "complejos de propiedades característicos de determinados tipos de lenguas, y que tales complejos deberían poder explicarse en términos de la elección de parámetros en uno u otro de los subsistemas" (1981 a, p. 6). Uno de los parámetros más estudiados en los últimos años es, por ejemplo, el de las lenguas que pueden omitir el sujeto (el denominado pro-drop parameter). Estas lenguas -el castellano y el italiano son ejemplos de ellas, frente al francés y el inglés - se caracterizan por admitir la libre inversión del sujeto, por la presencia de pronombres redundantes vacíos en las oraciones incrustadas, ('El chico que él dice que lo saludaste') por la violación aparente del filtro que-t y por otras propiedades (cfr. Chomsky, 1981 a, 4.3.) que están sistemáticamente ausentes en las lenguas en las que el sujeto no puede omitirse.

1.3. La tercera distinción que deseo considerar en esta introducción conceptual a la formulación actual de la lingüistica generativa es la oposición entre teorías derivacionales y teorías representacionales. Como señala Koster (1981) el paradigma clásico de esta teoría lingüística es, en esencia, un paradigma derivacional, centrado, por tanto, en la especificación de reglas transformatorias y de estructura sintagmática y en las interacciones entre ellas. Se ha indicado innumerables veces que el cometido fundamental de un modelo de descripción así concebido es representar la noción intuitiva de "relación" entre oraciones. Este paradigma supone, pues - como es también bien sabido- que oraciones del tipo de Le dio el libro a Juan frente a El libro le fue dado a Juan tienen la misma estructura profunda si bien difieren en su derivación. Frente al modelo clásico, el paradigma actual suele denominarse representacional debido a que en él, dicho en términos algo informales, las complejas derivaciones motivadas por las también complejas reglas transformatorias han sido sustituidas por un conjunto reducido, que puede llegar a ser nulo, de reglas muy simples a las que se añaden ahora filtros y condiciones de buena formación (condiciones sobre la representación) que se aplican a las estructuras profundas y a las estructuras superficiales.

En realidad, este enfoque representacional tiene su origen ya en 1971 en el trabajo tan conocido de Perlmutter sobre el orden de los clíticos y una consecuencia importante de su adopción, como me ha hecho notar Víctor Sánchez de Zavala, es que al abandonarse el enfo- 
que derivacional ya no se posee un formalismo único que constituya indisolublemente las reglas de buena formación de "fórmulas" y las de obtención de "teoremas", sino las dos cosas por separado. Lo cual podría indicar que también se ha dejado de lado (en la medida en que la anterior afirmación sea correcta) el formalismo de las funciones recursivas que permitía afirmar a Chomsky en 1955 y 1956 que el formalismo no va a la zaga,de la teoría sino al frente de ella.

Dentro de este enfoque representacional, entonces, las oraciones activa y pasiva que hemos mencionado más arriba pasan a tener la misma estructura - la misma representación- en todos los niveles significativos. Esta identidad de representación, esta ausencia de diferencias derivacionales, no es una mera contingencia dentro de la teoría de la rección y el ligamiento sino una necesidad teórica determinada por la teoría de la huella y por el "principio de proyección». Este principio estipula, en efecto, que el marco de subcategorización de un determinado elemento debe estar categorialmente representado en todos los niveles sintácticos (EP, ES y FL). Por consiguiente, tal principio obliga a postular elementos nulos o categorias vacías (elementos que pueden tanto generarse en la base, el caso de $P R O$, como ser producto de reglas de movimiento: las huellas que dejan éstas al actuar) que son centrales en el tipo de análisis sintáctico que se pretende llevar a cabo en este momento.

Conviene señalar también que el objetivo de un paradigma representacional es menos el de caracterizar la noción intuitiva de relación entre oraciones y más el de dar razón de dependencias interpretativas, fundamentalmente entre categorias léxicas, categorias vacías y elementos anafóricos. Los tipos de procesos formales que formarian parte, pues, de la facultad del lenguaje son diversos de los postulados anteriormente, y corresponde a una teoría que se ocupe de los correlatos psicológicos de la lingüistica formal el decir la última palabra acerca de cuál de los dos enfoques es más adecuado desde su punto de vista.

En este modelo sintáctico representacional, por último, resulta necesario introducir nuevos conceptos teóricos que o bien ni siquiera aparecian en el marco teórico anterior, bien se redefinen y adquieren una significación distinta en el actual. De entre ellos posiblemente las más relevantes sean las nociones de rección o gobierno y localidad, por una parte, y la de modularidad por otra.

Dicho brevemente, podemos retrotraer la noción de rección o gobierno al supuesto de la gramática tradicional y estructural de que ciertos elementos «dependen" de otros: así, por ejemplo, los complementos son dependientes de sus núcleos. No obstante, en el marco al que estamos haciendo referencia se proponen una definición estricta y 
una aplicación constreñida de dicha noción. La idea de localidad constituye un requisito que debe ser satisfecho por ciertas reglas de la gramática: las de movimiento, que sólo pueden operar dentro de límites bien definidos.

El descubrimiento importante que está también en la base de la introducción de estas nociones, y que ha sido extensamente corroborado en la investigación generativista, es el de que ciertas propiedades estructurales de la configuración $\mathrm{O}$ (=oración) intervienen en la caracterización y explicación de fenómenos oracionales de niveles diversos: sintácticos, morfológicos, semánticos e incluso pragmáticos. Asi, por ejemplo, el principio del gobierno, al que antes he aludido, el de mando de constituyente a él incorporado y la noción de dominio, actúan tanto en la explicación de un hecho sintáctico-morfológico: cómo se asigna caso abstracto a los NN con matriz fonológica, como en la justificación última de ciertos procesos de interpretación semántica: el funcionamiento de las reglas relacionadas con la correferencia, así como en la caracterización de fenómenos relacionados con el discurso: el de la distribución de temas y remas, por ejemplo. Volveremos sobre estas cuestiones en la segunda parte de este trabajo. (Pero cfr. Reinhart, 1983). La localidad, por otra parte, es fructífera tanto en el dominio morfológico de la concordancia como en el de la actuación de las reglas transformatorias de movimientos (cfr. Koster, 1978).

La noción de modularidad implica, si la caracterizamos primero por lo que no es, el rechazo de la idea de que la descripción de un fenómeno lingüistico sea simplemente un proceso de formalización constituido por reglas sistemáticamente ordenadas y por la justificación y extensión de las mismas. Por el contrario, se piensa hoy que la explicación de los hechos del lenguaje da lugar a un sistema complejo producido por la interacción de un grupo reducido de módulos o sistemas de principios que son en sí mismos relativamente simples. Para volver al ejemplo de la pasiva (cfr. a este respecto Demonte, 1983 a), un estudio exhaustivo de esta construcción obliga hoy a tener en cuenta la acción simultánea de las teorías del caso y de los papeles temáticos para entender parte de sus propiedades, no menos que la intervención de la regla 'mover $\alpha$ ' y del "principio de las categorias vacias». Los problemas diversos relacionados con el orden de las palabras en la frase parece que también podrian tratarse adecuadamente si se adopta una perspectiva modular. 


\section{ALGUNAS OBSERVACIONES SOBRE SINTAXIS Y DISCURSO}

2.1. Las consideraciones que hasta aquí hemos hecho se refieren a nociones teóricas de reciente incorporación o redefinición por la gramática generativa, y que tienen un papel fundamental en la estructuración de la teoría de la rección y el ligamiento. No obstante, el inventario de nuevas distinciones no se agota en ellas. En algunos de los primeros trabajos correspondientes al período de la teoría estándar ampliada (Chomsky 1975 a, 1975 b, 1976), en especial en los apartados que conciernen a las reglas posibles de interpretación semántica, Chomsky enuncia explícitamente (por vez primera) el alcance oracional del estudio permitido por su teoría lingüística y distingue claramente, en consonancia con otras reflexiones que se están produciendo simultáneamente en el ámbito del estudio del lenguaje, la gramática de la oración de la gramática del discurso. Como es evidente, y a diferencia de lo que sucede con las nociones que acabamos de reseñar, al señalar tal oposición entre estas dos subáreas de la ciencia lingüística Chomsky está más recordando cuáles son los límites de su propio territorio que incorporando al mismo nuevos problemas, o nuevas maneras de abordarIos; aunque esta segunda observación pueda merecer alguna cualificación como veremos inmediatamente.

En una formulación breve y esquemática podemos decir que, en el entendimiento de la teoría generativa, la gramática de la oración caracteriza las propiedades formales de la estructura constituyente de la oración y delimita la "forma lógica" de la misma, esto es, explica las propiedades semánticas de las oraciones que están exclusivamente determinadas por sus características estructurales, por la sintaxis. La gramática que se ocupa de las estructuras que trascienden el límite de la oración está constituida por las reglas y principios que establecen las condiciones para el empleo apropiado de las oraciones, o las intenciones y propósitos con que se las emplea. En Chomsky (1980 a) se sugiere asimismo que esta gramática no oracional sería la encargada de relacionar la gramática formal con otros sistemas de conocimiento y creencia, o con factores sociales que no forman parte del sistema abstracto de la gramática oracional (cfr. también Demonte, 1981 ap. 2.1.). A modo de ejemplificación de los elementos de ambas gramáticas, obsérvese que la regla o principio de control del sujeto de la completiva en una oración como

(1) Juan me prometió [PRO despedirse de ti] que establece que con un verbo como prometer PRO está controlado 
por el sujeto de la matriz, mientras que con un verbo como ordenar el que lo controla es el objeto de dicho verbo:

(2) Juan me ordenó [PRO despedirme de ti]

es una regla de la gramática de la oración puesto que la determinación de estas relaciones semánticas es por completo independiente del uso de estas oraciones en cualesquiera contextos 0 de las intenciones, conocimientos o creencias de los sujetos. Por el contrario, la regla o principio que establezca el significado del elemento pronominal en una oración como

(3) El llegará mañana

no puede pertenecer a la gramática oracional ya que tal significado sólo puede establecerse en el contexto del discurso. Debe recordarse, sin embargo, que la pronominalización y la referencia pronominal le conciernen también a la gramática oracional ya que es parte de su cometido el determinar por qué el pronominal y el $\mathrm{SN}$ subrayados puede ser correferenciales en (4a) pero no en (4b):

(4) a. Mi hermana dijo que ella no se humillaría ante él.

b. Ella dijo que mi hermana no se humillaria ante él.

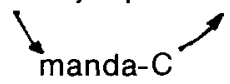

La explicación clásica, debida a Langacker (1969) apela a la regla de pronominalización que indica que un pronombre no puede preceder y mandar a su antecedente (un elemento A manda a B si el primer nudo $O$ que domina a A domina también a B). No obstante, la regla de Langacker hace predicciones falsas ya que hay oraciones como

(5) a. Cerca de él, Luis vio una araña horrible.

b. Con su coche, Maria puede hacer lo que quiere en las que la correferencialidad no se excluye a pesar de que el pronombre manda y precede a su antecedente. Por eso Reinhart (1976) va a proponer una alternativa a la regla de pronominalización de Langacker introduciendo la noción estructural de mando de constituyente (mando - C). (Un elemento A manda - $C$ a un elemento $B$ si el primer nudo ramificado que domina a $A$ domina también a $B$ ). Adoptando este principio estructural se puede formular una regla de asignación de correferencia como (6) (de Reinhart, 1976).

(6) Un pronombre no puede ser correferencial con un SN pleno sobre el cual él tenga mando-C.

(4b), por ejemplo, es una estructura que ratifica la regla (6). Volviendo a nuestras anteriores precisiones, esta regla (6) es un ejemplar típico de una gramática oracional, pues explica cómo una propiedad semántica - por lo menos semántica en un nivel intuitivo- se basa pura y exclu- 
sivamente en una relación estructural, en una propiedad de la configuración jerárquica de constituyentes, y no en factores como los que explican la construcción aparentemente similar de (3).

2.2. De lo que he indicado hasta aqui se sigue, asimismo, que el estudio y la delimitación del contenido empírico de los dos tipos de teorias del lenguaje antes definidos puede llevarse a cabo a través de una doble vía. Se puede considerar, en efecto, en qué divergen ambas perspectivas, en qué no coinciden ni tienen por qué coincidir, o se pueden estudiar las intersecciones e interrelaciones entre ambas. El primer camino es sin duda el más fascinante y el más prometedor pero de él no me ocuparé aquí porque excedería con mucho los objetivos de este trabajo (pero cfr. Demonte, 1981, ap. 2.1). Quiero decir, con otras palabras que se ha mostrado ya suficientemente en la literatura que se puede hacer gramática supraoracional con una gran independencia metodológica y conceptual de la gramática oracional. La segunda vía, más modesta y mucho más constreñida, es, en cambio, la que me interesa ilustrar. ¿Por qué mi interés por estudiar las zonas comunes a la oración y al discurso? En primer lugar, porque ello ha sido objeto de consideración por algunos generativistas y hay por lo tanto propuestas concretas que pueden someterse a contrastación; en segundo lugar, porque constituye un terreno interesante para la corroboración empírica de algunos de los niveles abstractos propuestos por la teoría actual, más concretamente, sirve para confirmar la existencia del así llamado nivel de la "forma lógica" o sintaxis de la semántica; y, en tercer lugar, porque esa comparación permite mostrar que la asignación de un determinado problema - pongamos por caso la cuestión de la tematización o topicalización- a uno u otro tipo de teoría gramatical no es una cuestión a priori sino algo que, en última instancia también está teóricamente determinado. Con otras palabras: un problema pertenecerá a una u otra teoría gramatical según cuál de ellas sea capaz de caracterizarlo y explicarlo más exahustivamente.

Vayamos, entonces, a la primera cuestión que acabo de sugerir, o sea, a la de si hay algún lugar de la descripción lingüística en que gramática oracional y gramática del discurso se engarcen de alguna manera y cómo es ese engarce.

2.2.1. Williams (1977): “Discourse and Logical Form", constituye, den- 
tro de la teoría generativa, un intento casi programático de abordar la cuestión antes señalada. Para comprender las aportaciones de Williams (1977) conviene recordar previamente que tanto la «teoría estándar ampliada" como la "teoría de la rección y el ligamiento", cuando reducen drásticamente la forma y la tipología de las reglas transformatorias que operan en el componente sintáctico (las reglas quedan reducidas a una única regla de movimiento: "Muévase $\alpha$ ), proponen que los procesos de elisión, antes concebidos como transformatorios, se realicen en el camino que va de la ES (estructura superficial) a la RF (representación fonética) (cfr. Chomsky 1980b). Williams va a mostrar, en primer lugar, que, en realidad algunos procesos de elisión pertenecen no a la gramática de la oración sino a la gramática del discurso y, por otra parte, que todas las estructuras con elisiones son, en última instancia, producto de reglas de interpretación semántica, tanto las que resultan de reglas oracionales como las que provienen de reglas del discurso.

Una segunda hipótesis central del programa de este lingüista es la de que los procesos de elisión propios del discurso se caracterizan porque no crean "formas lógicas» sino que se aplican a formas lógicas. Ahora bien, si esta suposición de Williams es verdadera, y puede corroborarse, tendremos nuevas pruebas a favor de la existencia de un nivel de análisis lingüístico, éste de la forma lógica, poco intuitivo a primera vista pero cargado de implicaciones para la concepción chomskiana de la adquisición del lenguaje.

En efecto, la existencia de este nivel que sustituye parcialmente al antiguo componente semántico interpretativo y en el que - por decirlo de una manera breve y aproximada - se construyen, a partir de las ES, configuraciones con cuantificadores y variables ligadas semejantes a las fórmulas de la lógica de primer orden, puede justificarse de varias maneras. Una primera justificación del mismo está en el hecho de que allí se procede a la desambiguación de las expresiones del lenguaje natural, pero quizá esta razón de ser no sea excesivamente interesante puesto que para sólo lograr tal fin no se requieren necesariamente los medios antes mencionados. Un segundo elemento crucial a favor de la existencia de dicho nivel proviene de la comprobación de que determinadas propiedades del lenguaje natural sólo pueden explicarse si se utilizan los conceptos y las configuraciones propios de este nivel. Razonar de manera siquiera aproximada esta segunda vía me llevaría muy lejos de lo que aquí persigo, pero permítaseme indicar al menos que gran parte de la más original investigación actual en gramática generativa está concentrada precisamente en ella. (Cfr., por ejemplo, Higginbothan, 1980; Huang, 1982; Rizzi, 1983). Se puede mostrar, en tercer lugar, que la FL existe si hace notar la necesidad de alguna «superficie 
intermedia que delimite la teoría enormemente restringida de la forma lingüística de la teoría más general de la semántica y la pragmática de los lenguajes naturales", como nos sugiere May (1977, pág. 2). Exactamente esta tercera cuestión es la que puede suscitarse si se amplía de una cierta manera el trabajo de Williams antes mencionado.

Situado, entonces, teóricamente el problema podemos volver a las elisiones oracionales y de discurso.

2.2.2. ¿Qué elisiones son propias del discurso y cuáles del nivel oracional? Si se establece como propiedad característica de las reglas de discurso el no estar limitadas a operar en el límite de una $O$ y no estar sometidas a condiciones sobre las reglas sintácticas como las de Ross (1967) o Chomsky (1973, 1976), entonces puede admitirse que las elisiones y sustituciones que tienen lugar en las siguientes secuencias son, en efecto, discursivas:

(7) a. ¿Dijiste que (Pedro) se llevó la bicicleta de Luis?

b. No, dije que $\left\{\begin{array}{l}\text { se llevó } \\ ? \varnothing \\ \text { no lo hizo }\end{array}\right\}$ la $\varnothing$ de María $\}$

(8) SN [EI hombre $_{0}$ [que se llevó la bicicleta de Luis] vio ${ }_{\mathrm{sN}}$ [al hombre ${ }_{0}$ [que $\left\{\left\{\begin{array}{l}\text { se llevó } \\ \varnothing_{\text {hizo lo mismo (con) la de Maria }}\end{array}\right\}\right.$ la $\varnothing$ de María

La elisión de NN y la sustitución de un SV por hacer (lo mismo) son -al menos a primera vista - procesos de discurso ya que, como muestran las oraciones de (7), pueden realizarse en el contexto de la conversación y no están constreñidos, como pone en evidencia (8), por la «restricción del SN complejo». ¿Cuál es la razón de la elisión no permitida de (7b) y (8)? A salvo de lo que indique un estudio más en profundidad, sugiero que en estas construcciones opera la regla de "vaciado" Igapping]. Esta regla, que - conforme a ciertas versiones de la misma-borra un $V$ cuando está flanqueado por dos «constituyentes» (cfr. Stillings, 1975), es, al menos en la gramática del inglés, una regla de elisión típicamente oracional que contrasta, por consiguiente, en este aspecto con la de "elisión-sustitución del SV por hacer (lo mismo)" -como acabamos de ver. Obsérvense también las expresiones de (9), 
(9) a. ¿Juan se fue a Dinamarca?

b. No, [Maria] $\varnothing \quad$ [a su casa]

que muestran, una vez más, que el vaciado produce oraciones marginales cuando se efectúa en el discurso, a diferencia de lo que sucede en un contexto oracional, como se ve en las oraciones de (10):

(10) a. Susana fue al cine y Esther $\varnothing$ a la discoteca.

b. Tu hermana compró los billetes del avión y su amigo $\varnothing$ las entradas para el Metropolitan.

c. El satélite está en la órbita correcta y el cohete $\varnothing$ en el océano Indico.

La mera prueba directa que proporcionan las oraciones de (9) y (10), sin embargo, no basta para determinar que hay elisiones oracionales -el "vaciado»- y elisiones de discurso, sino que es necesario proceder a un análisis algo más exhaustivo y complejo.

Lo primero que quiero poner de relieve es que hay datos relevantes que permiten sugerir que el "vaciado» debe ser 'anterior' —en algún sentido de 'anterior' - a "elisión de NN» y a "sustitución del SV», las dos reglas hipotéticas de discurso que estamos considerando. Observemos las oraciones de (11) y (12):

(11) a. María compró la casa y Daniel compró el chalet. b. María compró la casa y Daniel $\varnothing$ el chalet.

(12) a. ¿Compró María la casa?

b. (i) -Sí, María compró la casa y Daniel Øel chalet.

(ii) -Sí, María lo hizo, y Daniel compró el chalet.

(iii) $^{*}$-Sí, María lo hizo, y Daniel $\varnothing$ el chalet.

En (11) la regla de "vaciado" se aplica en un dominio oracional y el resultado es la oración gramatical (11b), semejante en todos los sentidos a (12b. i). En (12 b.ii.) se aplica, a través del límite oracional, la regla de «sustitución del SV" y, de nuevo, el resultado es gramatical. ¿Por qué es agramatical, entonces, (12b.iii) donde deben haberse aplicado tanto "vaciado" como "elisión del SV"? Una manera de predecir esta agramaticalidad es suponer, como E. Williams, que la regla de «vaciado" es, en realidad, una regla interpretativa que establece una relación anafórica entre un $V$ ocupado por un elemento $\Delta y$ otro verbo situado en un coordinando precedente en una estructura como la de (13):

(13) María ${ }_{s v}\left[\right.$ [hizo $_{\mathrm{sN}}[\mathrm{lo}]$ y Enrique $\mathrm{v}[\Delta]$ el chalet.

Como el verbo comprar ha sido eliminado por la regla de sustitución 
por hacer, la interpretación del elemento $\Delta$ no podrá llevarse a cabo de la manera como se hace en (12b.i.).

Ahora bien, si "vaciado no fuese una regla interpretativa sino un proceso de elisión en sentido estricto, esto es, una regla que hace desaparecer material fonológico por identidad con otro elemento de la configuración en que se encuentra, entonces la agramaticalidad de (12b.iii) podría predecirse indicando que "vaciado" se aplica después de «sustitución por hacer», o sea, en el orden contrario al que acabamos de sugerir. El efecto de esta consideración no es sólo el de situarnos frente a una paradoja, más importante aún: tal suposición implica que una regla de discurso debería aplicarse antes que una regla oracional. Veremos inmediatamente, sin embargo, que hay otros elementos de juicio importantes a favor de mantener el supuesto básico de Williams de que ląs reglas oracionales deben preceder a las discursivas.

2.2.3. Como he sugerido anteriormente, en las lenguas naturales parecen darse propiedades que sólo podrían explicarse si se concibe algún nivel descriptivo en el cual operen expresiones con operadores o cuantificadores y variables ligadas por ellos. En efecto, un tipo de construcción que parece reunir características que hacen pertinente la postulación de un nivel tal son las denominadas de "cuantificación inversa" (cfr. May, 1977 y Huang, 1982) como las que aparecen en (14):

(14) a. Muchos hombres aman a tres mujeres.

b. Muchas personas han grabado cuatro sinfonias.

Las oraciones de (14) son ambiguas, por lo que al alcance de las expresiones cuantificadas respecta. Cada una de ellas admite una lectura en la cual los cuantificadores están en el mismo orden en el que se encuentran en la ES y otra en la cual aparecen en el orden inverso. Así, por ejemplo, (14a) puede significar bien que existen muchos hombres, que pueden singularizarse, tal que ellos aman a tres mujeres, cualesquiera que éstas sean; bien que existen tres mujeres determinadas (Diana, Irene y Luisa) tal que muchos hombres indeterminados las aman. La primera interpretación se representa en (15) donde el cuantificador muchos tiene un alcance más ancho que tres, la segunda en (16) donde el cuantificador más ancho o amplio, que incluye al otro, es tres. (En (15) y (16) sigo la representación de May, 1977).

(15) $o_{1}\left[\right.$ [muchos hombres $\alpha \propto{ }_{o_{2}}$ [ [tres mujeres] $\beta \quad{ }_{\mathrm{o}_{3}}[\alpha$ ama (a) $\beta] l]$

(16) ${ }_{o_{1}}\left[\right.$ [tres mujeres] $\beta_{o_{2}}\left[\right.$ [muchos hombres] $\alpha{ }_{{ }_{0} 3}[\alpha$ ama (a) $\left.\left.\beta]\right]\right]$ 
La suposición generalmente admitida es que esta ambigüedad de alcance sólo puede detectarse y caracterizarse en la FL. Así, May (1977) concibe una regla de "ascenso del cuantificador» que adjunta un cuantificador en $O$ y que está constreñida por ciertas condiciones sobre la forma lógica. Es esta regla precisamente la que forma (15) y (16) a partir de la estructura $S$ (14a). Un análisis semejante puede proponerse para (14b).

Lo que quiero considerar ahora, aunque sea brevemente, es la relación que existe entre esta regla del nivel FL y procesos de elisión como los que hemos estado discutiendo. Esa relación se puede esquematizar diciendo que, por extraño que parezca, la ambigü edad en la interpretación del alcance de los cuantificadores se mantiene en el discurso mientras que las dos interpretaciones pueden reducirse a una en oraciones simples coordinadas donde se haya producido "vaciado". Consideremos las oraciones (17) y (18):

(17) a. ¿Muchas personas tienen grabadas cuatro sinfonías de Mahler?

b. No, muchas $\varnothing$ tienen grabadas cuatro $\varnothing$ de Beethoven. b'. *No, muchas personas $\varnothing$ cuatro sinfonias de Beethoven.

(18) a. Muchas personas tienen grabadas cuatro sinfonías de Mahler y muchas $\left\{\begin{array}{c}\varnothing \\ \text { personas }\end{array}\right\}$ cuatro $\left\{\begin{array}{c}\varnothing \\ \text { sinfonias }\end{array}\right\}$ de Beethoven.

b. Muchos hombres aman a tres mujeres de Madrid y muchos $\left\{\begin{array}{c}\varnothing \\ \text { hombres }\end{array}\right\}$ a tres $\left\{\begin{array}{c}\varnothing \\ \text { mujeres }\end{array}\right\}$ de Barcelona.

En (17) la elisión de $\mathrm{NN}$ se produce contando con los antecedentes emitidos en la oración anterior en el texto. En ese caso no es posible el vaciado, como ya hemos indicado y como muestra (17b'). En (18a) y (18b), oraciones en las que sí tiene lugar el vaciado, no es posible entender sino que se trata de cuatro sinfonías indeterminadas o de cuatro mujeres indefinidas; desaparece, por lo tanto, la interpretación (16). Véase también (19) que corrobora este juicio y que deja más claro que esa desaparición no es imputable a las elisiones de SSNN:

(19) Muchos austriacos tienen grabadas cuatro sinfonias de Mahler y muchos alemanes $\varnothing$ cuatro sonatas de Beethoven. 
¿Cuáles son las implicaciones de los hechos que acabamos de poner en evidencia? Obsérvese, como cuestión previa, que esta comprobación contradice, en cierto modo, la idea de sentido común de que el discurso puede permitir con especial flexibilidad el empleo de expresiones vagas por disponer de un cúmulo mayor de elementos para efectuar desambiguaciones. Si ello fuera así, cabría esperar que la respuesta (17b) no fuese ya ambigua y, sin embargo, sigue siéndolo. Más importante aún es el hecho de que esta distribución de las interpretaciones lógicas se puede predecir sin dificultades si se adopta la hipótesis de Williams que aquí hemos defendido. En efecto, si las reglas de discurso no crean formas lógicas sino que se aplican a ellas, como antes he recordado, entonces debe esperarse tanto que no se produzcan cambios en las interpretaciones lógicas cuando tales formas están ya delimitadas, como que pueda haberlos en el proceso de construcción de dichas formas lógicas. Por eso, el vaciado del verbo se asocia con la reducción de la ambigüedad, mientras que la elisión de NN, que es un proceso ulterior según nuestra hipótesis, ya no lo permite. Con otras palabras, mientras no se ha concluido el proceso de elaboración de las FFLL pueden todavía producirse cambios, una vez determinadas, los cambios no serian ya posibles. Este hecho indica también, por último, que los dos tipos de elisiones que estamos considerando no se producen simultáneamente sino que están separadas por esa especie de «superficie intermedia" que limita el territorio de la forma lingüística del de la semántica y la pragmática conectadas con los elementos exteriores al lenguaje.

2.3. Como señalé al comienzo de esta segunda parte, otra manera de estudiar la relación entre los dos tipos de gramática que estamos considerando consiste en preguntarse si alguna de ellas ha aportado nuevas luces acerca de algún fenómeno considerado en principio como peculiar del ámbito de la otra. En este sentido, numerosos autores han puesto de relieve, por ejemplo, la dificultad de agotar en la gramática oracional el estudio de las propiedades de las articulaciones en tema y rema, (aunque el descubrimiento de esta estructuración -o perspectiva funcional de la oración- se deba a un modelo de gramática oracional: la nueva escuela de Praga). Un aspecto de estas estructuras que parece difícil de resolver si no se adopta la perspectiva del discurso es el del hallazgo o identificación del tema, como ha sido puesto de relieve por numerosísimos autores.

La observación anterior es obviamente cierta. El «tema» o «informa- 
ción conocida" o, mejor, "aquello de lo que la oración trata pragmáticamente» [pragmatic aboutness (cfr. Reinhart, 1982)] es una relación pragmática que, por consiguiente, sólo puede determinarse completamente recurriendo al contexto de la emisión. No obstante, como es también sabido, no son sólo factores de esta naturaleza los que operan en las articulaciones tema-rema. Así, por ejemplo, se ha mostrado que en algunos casos la elección de tema está constreñida asimismo por propiedades semánticas. Es muy difícil, y a veces imposibie, pongamos por caso, que los SS.NN., cuantificados, cuando no son referenciales, se interpreten como temas.

De manera similar, hay consideraciones de tipo estructural o sintáctico que inciden en la elección del tema o en la determinación de qué es un tema posible para una determinada oración. Estas razones, por otra parte, son dignas de ser tenidas en cuenta puesto que el estudio de la tematización en el terreno del texto - por ejemplo, los trabajos sobre "progresión temática" propuestos por Daneš- parece que no podría prescindir del señalamiento del tema y el rema en cada una de las oraciones nucleares. De entre tales consideraciones, las más relevantes y de tratamiento más extendido en la literatura son las que me limitaré a esbozar en los subapartados que siguen.

2.3.1. Existe la tendencia a colocar el tema en la posición de sujeto $y$, paralelamente, a identificar el sujeto con el tema oracional. No es necesario insistir, creo, en que ésta no es más que una generalización estadística y que, como es evidente, hay numerosas ocasiones en las cuales el tema y el sujeto no coinciden. En la siguiente secuencia de oraciones de un texto de A. Carpentier (La consagración de la primavera), por ejemplo, el tema de la segunda y tercera oración es, indudablemente, la danza folklórica, introducido en la primera, pero el desarrollo de la exposición permite representarlo como/a. Lo que resultaría anómalo, sin embargo, seria una secuencia como la de (20b) donde un elemento no esperado irrumpe bruscamente como tema en una posición marcada para ello, como veremos inmediatamente.

(20) a. "La danza fo-lórica, vista en su ambiente, es magnífica. Pero la llevas a un escenario y te resulta larga, repetida, monótona. Para encaramarla en un teatro hay que repintarla, encuadrarla, ponerla en condiciones de que se le echen los focos encima" (op. cit. pág. 257, siglo XXI. Eds., cuarta ed.: 1979). 
b. La danza folklórica, vista en su ambiente, es magnífica. En cuanto a Isolda, déjala que cante.

2.3.2. Ahora bien, la investigación sintáctica ha puesto de manifiesto que hay ciertas configuraciones que tienen posiciones fijas, marcadas, para el tema y que, por consiguiente, los constituyentes que se colocan en esas posiciones son necesariamente, temas. Tal es el caso de las estructuras con dislocación izquierda del tipo de las de (21):

(21) (En cuanto a) Tu padre, dice María que no se le ha visto un detalle.

Estas estructuras, como ha sido señalado por Bernárdez (1982), son semejantes a las de aquellas lenguas que, por ejemplo, destacan el elemento temático por medio de sufijos específicos, como en el caso de quechua o del japonés.

La dislocación izquierda ha recibido extenso tratamiento en la literatura gramatical reciente e incluso hay estudios específicos dentro de la gramática generativa que emplea datos del castellano (cfr. Rivero, 1980). El análisis actual de estas construcciones — distinto del de Ross (1967)— propone, siguiendo a Chomsky (1977), que estas estructuras no se generen por medio de transformaciones sino directamente en la base. En las oraciones dislocadas, entonces, se genera un constituyente en la posición TEM (que es una posición superior a la de la $\bar{O}$ que domina al resto de la oración) en una estructura como (22). Hay reglas interpretativas ulteriores que establecen una relación anafórica entre este Tema y otro elemento de la cadena oracional,

(22) $\bar{c}$ tem[ $\left.x_{i}\right] \bar{o}[$ compl $\left.\left.\left.] d \ldots j_{i i} \ldots\right]\right]\right]$

Para comprender más cabalmente la naturaleza de estas construcciones dislocadas se las debe comparar con las estructuras semejantes, aunque no idénticas, de las oraciones utematizadas" como (23):

(23) Noticias, dice Pedro que tiene.

Estas oraciones, por diversas razones y propiedades que no es pertinente comentar en este trabajo (pero cfr. Rivero, 1980), se generan en cambio por medio de transformaciones y dan lugar a una estructura derivada como la de (24):

(24) ol [TEMA $\left.\left.]_{\text {comp }} \mathrm{d}[\ldots]\right]\right]$

La diferencia estructural fundamental, a nuestros efectos, entre ambas oraciones consiste en que en las construcciones con dislocación izquierda el sujeto no es nunca núcleo (o cabeza) dominante (cfr. Reinhart, 1983). Con otras palabras, el sujeto no tiene mando-C sobre el 
constituyente colocado en la posición inicial y, paralelemente, el único nudo que tiene mando- $C$ sobre todos los otros elementos del dominio de la $O$ completa es el sintagma dislocado. Estas propiedades, sin embargo, no se dan en las estructuras tematizadas. En efecto, si - como propone Reinhart (1983), ap. 1.2.2.-, la noción de mando-C se reformula de modo que pueda dar cuenta del hecho de que COMP forma parte del dominio del sujeto de $\mathrm{O}$ en $\overline{\mathrm{O}}$, entonces podrá decirse que el sujeto de (23) tiene mando-C sobre el elemento tematizado. Otra diferencia importante estriba en que mientras en las dislocaciones el elemento antepuesto es el Tema - como ya se ha indicado- en las tematizadas pueden ser Temas tanto el SN antepuesto como el sujeto. Más aún, como han señalado algunos investigadores, los SSNN antepuestos por medio de la regla de tematización pueden recibir entonación de foco, en cuyo caso no funcionan como Tema, como es evidente. Faltando esa entonación característica, repitamos, pueden ser temas tanto el elemento inicial como el sujeto de la oración. Esta diferente función de los dos tipos de elementos antepuestos puede contrastarse con bastante facilidad si se formula una interrogación con entonación normal. Por regla general, aquello por lo que se pregunta constituye la información nueva. Obsérvense (25) y (26) correspondientes a 21) y (23), RESPECTIVAMENTE:

(25) a. ¿Dice María que a tu padre no se le ha visto un detalle?

b. Respuesta poco posible: -No, dice que a tu madre.

c. Respuesta normal: - No, dice que ha estado fenomenal.

(26) a. ¿Noticias dice Pedro que tiene?

b. Respuesta poco posible: -No, lo dice María.

c. Respuesta normal: -No, dice que no tiene nada.

A juzgar por estas respuestas el tema en $(26)(=23)$ no es noticias (el elemento tematizado) sino Pedro (el sujeto oracional), por eso resulta extraña una contestación como (26b), que alude a la información conocida. (26c) muestra que noticias es, en realidad, el rema. En (25) el elemento desplazado si es la información compartida y de ahí lo inesperado de la respuesta (25b).

¿Cómo se relacionan las propiedades estructurales a que antes aludíamos con la existencia o ausencia de posiciones fijas para el Tema? Reinhart (1983) plantea la posibilidad de que la tematicidad sea una relación de primacía y que esta relación de primacia o superioridad pragmática estaría fuertemente relacionada con la de núcleo o cabeza de un dominio $[D(o m a i n)$ head] donde aun nudo $A$ es un núcleo $\mathrm{D}$ (ominante) de un dominio $\alpha$ si y sólo si $A$ manda- $C$ a todos y solamente los 
nudos de $\alpha$ ". Así las cosas, los hechos que estamos considerando se explican adecuadamente si se tiene en cuenta que en las oraciones tematizadas el núcleo dominante es el sujeto y, por tanto, es el que tiene primacía pragmática; de manera similar, en las estructuras dislocadas, como el constituyente antepuesto es el único que tiene mando- $C$ sobre todos los elementos de la oración, es el que inequivocamente se constituye en Tema oracional.

Lo interesante de esta comprobación, por último, no es sólo el que caracterice a una estructura congelada - fija - de tema-rema y prediga tendencias en otras configuraciones sino que también permite determinar la estructura de tales articulaciones en otros contextos, por ejemplo, en las oraciones con sintagmas preposicionales antepuestos. En efecto, esta hipótesis anticipa que los SSPP que sean adverbiales oracionales deberán ser temas cuando aparezcan en posición inicial a diferencia, pongamos por caso, de los SSPP adverbiales del SV que no podrán ostentar tal primacía pragmática porque tampoco poseerán dominio estructural. No voy a considerar aquí ejemplos de ambos tipos de SSPP pero remito al lector a Reinhart (1983).

A lo largo de estas páginas, en síntesis, tras introducir algunos conceptos claves en el marco teórico actual, he intentado precisar de varias maneras cómo se plantea la relación sintaxis de la oración-sintaxis del discurso en la constreñida perspectiva de la gramática generativa. Lo que este trabajo proporciona, en última instancia, son dos escuetos argumentos que muestran, de una parte, que puede haber un estrato de la descripción lingüística en el que dejen de aplicarse las reglas de una gramática y comiencen a aplicarse los de la otra y, de otra, que un problema de índole indudablemente pragmática puede explicarse, al menos parcialmente, si se recurre a principios estructurales que operan en la gramática de la oración. No es esperable ninguna biunivocidad sintaxis-discurso o sintaxis-pragmática, si son correctos los supuestos básicos de la lingüística chomskiana, sino precisamente esto; correlaciones parciales e incidencia de propiedades estructurales en fenómenos de índole no meramente estructural. 


\section{REFERENCIAS BIBLIOGRÁFICAS}

BERNARDEZ, E. (1982) Introducción a la lingüística del texto. Madrid, Espasa Calpe, 1982.

BOTHA, R. (1982) "On 'the Galilean style' of linguistic inquiry". Lingua $58,1-50$.

CINQUE, G. (1982) «On the theory of relative clauses and markedness».

The linguistic review 1:3, 247-296.

COMRIE, B. (1981) Language universals and linguistic typology. Oxford: Basil Blackwel.

CHOMSKY, N. (1965) Aspects of the theory of syntax. Cambridge: MIT

Press. [Trad. cast. Aspectos de la teoría de la sintaxis. Madrid: Aguilar, 1970].

-11973) “Conditions on transformations". En: S. Anderson y P. Kiparsky (eds.) A. Festchrift for Morris Halle. Nueva York: Holt, 232286. [Trad. cast.: “Condiciones sobre las transformaciones». En. N. Chomsky: Ensayos sobre la forma y la interpretación, Madrid: Cátedra, 1982, 187-239].

-(1975a) Reflections on language. Nueva York: Pantheon. [Trad. cast.:

Reflexiones sobre el lenguaje. Barcelona: Ariel, 1979].

-(1975b) "Questions of form and interpretation». Linguistic analysis

1:1, 75-109. [trad. cast.: Cuestiones de forma y de interpretación.

Cuadernos Teorema. Valencia: Revista Teorema, 1977].

-(1976) "Conditions on rules of grammar". Linguistic analysis 2:4, 
303-352. [Trad. cast. "Condiciones sobre las reglas de la gramática». En: N. Chomsky: Ensayos sobre la forma y la interpretación. Madrid: Cátedra, 1982, 95-185].

CHOMSKY, N. (1977) "On wh-movement". En P. Culicover, T. Wasow y A. Akmajian (eds.). Formal syntax. Nueva York: Academic Press, 71-132.

-(1980a) Rules and representations. Nueva York: Columbia University Press.

- (1980b) "On binding" Linguistic Inquiry 11: 1;1-46.

-(1981a) Lectures on government and binding. Dordrecht: Foris.

-(1981b) «Markedness and core grammar». En: A. Belletti, L. Brandi

y L. Rizzi (eds.) Theory of markedness in generative grammar.

Pisa: Scuola Normale Superiore, 123-146.

-1982) Some concepts and consequences of the theory of government and binding. Cambridge: MIT Press (Linguistic Inquiry Monographs).

DANES, F. (1974) Papers on functional sentence perspective. La Haya: Mouton.

DEMONTE, V. (1981) «Forma lógica y sintaxis autónoma; la teoría sintáctica en la actualidad" Teorema XI, 2-3, 135-166.

-(1983a) «Pasivas léxicas y pasivas sintácticas en español». En: Serta Philologica F. Lázaro Carreter, vol. I, Madrid: Cátedra, 141-157.

-(1983b) «Principios y representaciones en sintaxis: la teoría de la rección y el ligamiento". Verba, en prensa.

HIGGINBOTHAM, J. (1980) «Pronouns and variables" Linguistic Inquiry 11:4, 679-708.

HUANG, CH. (1982) Logical relations in chinese and the theory of grammar. Tesis doctoral inédita. MIT.

KAYNE, R. (1983) “Connectedness». Linguistic inquiry 14: 2, 223-250.

KOSTER, J. (1978) Locality principles in syntax. Dordrecht: Foris.

-(1981) “Configurational grammar». En R. May y J. Koster (eds.) Levels of syntactic representation. Dordrecht: Foris, 185-205.

LANGACKER, R. (1969) "On pronominalization and the chain of command". En: D. Reibel y S. Schane (eds.) Modern Studies in English. Englewood Cliffs: Prentice Hall, 160-186.

MAY, R. (1977) The grammar of quantification. Tesis doctoral inédita. MIT.

MILNER, J.C. (1982) «Pour un usage du concept de marque en syntaxe comparative». En: Ordres et raisons de langue. París: Seuil, 126180.

PERLMUTTER, D. (1971) Deep and surface structure constraints in syntax. Nueva York: Holt. 
REINHART, T. (1976) The syntactic domain of anaphora. Tesis doctoral inédita. MIT.

-(1982) "Pragmatics and linguistics: An analysis of sentence topics". Mimeografiado por el IULC.

-(1983) Anaphora and semantic interpretation. Londres: Croom Helm. RIZZI, L. (1982) Issues in Italian syntax. Dordrecht: Foris.

-1983) "On chain formation». Presentado en la reunión anual de la GLOW. York, marzo de 1983.

ROSS, J. (1967) Constraints on variables in syntax. Tesis doctoral inédita. MIT.

RIVERO, M.L. (1980) "On left-dislocation and topicalization in Spanish». Linguistic Inquiry 11: 2, 363-394.

STILLINGS, J. (1975) "The formulation of gapping in English as evidence for variable types in syntactic transformations». Linguistic Analysis, 1:3, 247, 274.

WILLIAMS, E. (1977) «Discourse and logical form». Linguistic Inquiry 8:1, 101-140. 